\title{
Utilização de imagens digitais para avaliação da coloração de banana prata submetida a diferentes tratamentos pós-colheita
}

\author{
Use of digital images for color evaluation in 'banana prata' \\ submitted to different post-harvest treatments
}

\author{
Simone Silmara Werner ${ }^{1 *}$; Silvia Renata Machado Coelho ${ }^{2}$; \\ Anna Paula Poncio ${ }^{3}$; Letícia Ferreira ${ }^{3}$
}

\begin{abstract}
Resumo
O presente trabalho objetivou avaliar mudança da coloração durante o armazenamento de banana prata submetida a diferentes tratamentos pós-colheita utilizando imagens digitais. Os frutos, com coloração da casca totalmente verde, foram submetidos a seis tratamentos: $\mathrm{T}$ - imersão em água destilada por 3 minutos (testemunha), $\mathrm{H} 3$ - hidrotérmico $\left(50^{\circ} \mathrm{C}\right.$ por 3 minutos), $\mathrm{H} 8$ - hidrotérmico $\left(50^{\circ} \mathrm{C}\right.$ por 8 minutos), $\mathrm{HP}$ - imersão em hipoclorito $0,2 \%$ por 3 minutos, OS - imersão em óleo de soja $10 \%$ por 3 minutos e OM - imersão em óleo mineral $10 \%$ por três minutos, sendo em seguida armazenados em condições ambientais durante 14 dias, avaliados nos períodos 1, 7 e 14 dias utilizando imagens digitais, e diariamente utilizando escala visual. Os frutos submetidos aos tratamentos OS e OM preservaram a coloração verde da casca por um tempo maior, mas ocorreram alterações na coloração normal devido a formação de pontos escuros, indicando possível inadequação dos tratamentos OS e OM. Os dados obtidos através das imagens digitais confirmaram as observações visuais, indicando relação entre os métodos.
\end{abstract}

Palavras-chave: $M u s a s p$, amadurecimento, cor da casca

\begin{abstract}
This trial aimed at analyzing the changes of banana "Prata" peel color through digital images, submitted to post harvest treatments in order to increase storage time. The fruits, whose peels color were totally green, were submitted to six different treatments: $\mathrm{T}$ - immersion in chlorine water for three minutes (control), $\mathrm{H} 3$ - hydrothermal $\left(50^{\circ} \mathrm{C}\right.$ for three minutes); $\mathrm{H} 8$ hydrothermal $\left(50^{\circ} \mathrm{C}\right.$ for eight minutes $) ; \mathrm{HP}$ - immersion in hypochlorite $0.2 \%$ for three minutes; OS - immersion in soybean oil at $10 \%$ for three minutes and $\mathrm{OM}$ - immersion in mineral oil at $10 \%$ for three minutes. The fruits were stored at room temperature and analyzed at 1, 7, 14 days after using digital images and also daily visual evaluation. The treatments OS and OM preserved the banana green color for a longer time, but there were some changes in the normal peel color due the formation of dark stain spots, indicating probable inadequate OS and OM treatments. The fruits submitted to H3, H8 and HP treatments ripenned faster, considering the observed color. Both visual and digital images confirmed the values obtained. Suggesting a method relationship.
\end{abstract}

Key words: Musa sp, ripening, peel color

1 Biológa, aluna do Curso de Pós-Graduação em Agronomia - UNIOESTE - Marechal Cândido Rondon - PR.

2 Engenheira Agrônoma, Doutora, Professor Adjunto do curso de Graduação e Pós-Graduação em Engenharia Agrícola - CCET UNIOESTE, Rua Universitária, 2069, Jd Universitário 85819-110 - Cascavel - PR. E-mail: srmcoelho@unioeste.br.

3 Bióloga, Universidade Paranaense - UNIPAR - Cascavel - PR. 


\section{Introdução}

A banana, cultivada em uma área superior a 4,45 milhões de hectares, em 127 países, com mais de 72,59 milhões de toneladas produzidas em 2005, é a fruta mais consumida no mundo. Altamente energética, possui quantidades significativas de sais minerais como Cálcio, Magnésio, Ferro, Potássio, Sódio e vitaminas, tais como: Retinol, Tiamina, Riboflavina, Niacina e Ácido Ascórbico (BORGES, 2003; FOOD AND AGRICULTURE ORGANIZATION OF THE UNITED NATIONS - FAO, 2006). O Brasil, segundo maior produtor (6,70 milhões de toneladas em 2005), é um pequeno exportador, limitado pelo alto consumo interno e pela falta de controle de qualidade do produto (BRACKMANN et al., 2005).

Os requisitos de qualidade estão relacionados muito mais com a aparência e distribuição, do que as característicasinternas, devendoapresentar-seisentos de sinais ou ataques de fungos e insetos, parasitas e desordens fisiológicas, livres de queimaduras, amassados ou manchas (MATSUURA; COSTA; FOLEGATTI, 2004; CHITARRA; CHITARRA, 2005). A qualidade de banana é influenciada pelo armazenamento e amadurecimento, sendo que vários fatores devem ser controlados visando uniformizar o amadurecimento e comercialização. Uma série de tratamentos que tem sido desenvolvida perante manuseio pós-colheita, para retardar o amadurecimento e aumentar a qualidade do fruto (BOTREL et al., 2002; CAMPOS; VALENTE, PEREIRA, 2003).

Para a comercialização, a aparência é o atributo mais importante, estando relacionada com características como tamanho, forma, coloração e presença de defeitos. A ausência de pintas pretas é uma das características mais relevantes, sendo fator decisivo para cerca de $70 \%$ dos consumidores (MATSUURA; COSTA; FOLEGATTI, 2004).

As cores variam em três dimensões: luminosidade (L), a qual representa a relação entre a luz refletida e a luz absorvida; tonalidade cromática ou coloração
(H), a qual é o aspecto da cor que pode ser descrito por palavras, e croma (C), o qual indica o quanto a cor difere do cinza (POMERANZ; MELOAN, 1994). Estas dimensões são arranjadas no espaço físico de diversas maneiras, de acordo com o sistema de cor. Vários sistemas tem sido criados para quantificação destas estimativas: CIE, Hunter e sistema Munsell. Estes sistemas foram padronizados e é possível, através de programas matemáticos, a interconversão dos dados entre todos os sistemas (SOARES, 2000).

O CIE, em 1971, definiu novo espaço de cor denominado CIELAB, criado pela transformação não linear do sistema CIE, definindo o espaço dentro das coordenadas retangulares $\left(L^{*}, a^{*}, b^{*}\right)$ junto com outro espaço nas coordenadas cilíndricas: $\mathrm{L}^{*}$ (luminosidade), $\mathrm{H}^{*}$ (tonalidade cromática ou coloração) , $C^{*}$ (croma ou saturação) (SOARES, 2000). A tonalidade cromática $\left(\mathrm{H}^{*}\right)$ é o atributo pelo qual se identificam as cores violeta, azul, amarelo laranja, vermelho e púrpura. A luminosidade $\left(\mathrm{L}^{*}\right)$ descreve a cor em termos de mais clara e mais escura (de preto a branco) e é expressa em uma escala de 0 a 100 , na qual o zero representa o preto absoluto e o 100 representa o branco absoluto. Já as coordenadas cartesianas a* representa o componente vermelho-verde e $b^{*}$, o componente amarelo-azul (ASSOCIAÇÃO BRASILEIRA DE NORMAS TÉCNICAS - ABNT, 1992).

A medição de parâmetros de cor, por meio de aparelhos específicos, apresenta custo elevado, porém, com a utilização de metodologia adequada é possível, com equipamentos de baixo custo, a obtenção de parâmetros de cor em alimentos. Além disso, produtos sem uniformidade, como no caso dos grãos, frutas e hortaliças, não apresentam boa repetibilidade nas medições de cor em aparelhos específicos (SACHS, 2002). Mendonza, Dejmek e Aguilera (2006), desenvolvendo um sistema computacional para quantificação de cor em frutos, sugeriu que o sistema L*, a*, b* é o melhor espaço de cor para quantificação em alimentos com superfícies curvas e os resultados devem ser interpretados com precaução, utilizando amostras controle. 
Sachs (2002) desenvolveu um aplicativo para ler, pixel a pixel, cores de área pré-selecionada de imagem digitalizada. A amostra de interesse pode ser digitalizada com utilização de "scaner" ou fotografada em condições padronizadas, gerando uma imagem digital. O programa desenvolvido converteria as áreas selecionadas das imagens em conjuntos de valores médios nas três cores básicas, vermelho, verde e azul (RGB), os quais podem ser convertidos para o sistema L*, a*, b* (SACHS et al., 2001).

Diante do exposto, o presente trabalho teve por objetivo avaliar as alterações na coloração da casca da banana prata submetida a diferentes tratamentos pós-colheita, utilizando imagens digitais e verificar se esta metodologia apresenta correlação com a avaliação visual.

\section{Material e Métodos}

\section{Amostras}

Utilizou-se frutos de bananas do cultivar "Prata", adquiridos no Centro de abastecimento de Cascavel (CEASA), provenientes de São Paulo. Após serem retirados das caixas, os frutos foram selecionados, descartando-se aqueles que estavam fora do padrão (BOTREL et al., 2002), sendo em seguida, aleatoriamente agrupados em trinta e seis parcelas, formadas por um buquê de três frutos cada.

\section{Tratamentos pós-colheita}

Os frutos foram submetidos a seis tratamentos: Testemunha com imersão em água destlidade à temperatura ambiente durante três minutos (T); imersão dos frutos em água clorada à temperatura de $50^{\circ} \mathrm{C}$ durante três minutos $(\mathrm{H} 3)$; imersão em água potável à temperatura de $50^{\circ} \mathrm{C}$ durante oito minutos (H8); imersão em hipoclorito de sódio a $0,2 \%$ de cloro ativo, por três minutos (HP); imersão em óleo de soja na concentração de $10 \%$ em água destilada durante três minutos (OS) e imersão em óleo mineral na concentração de $10 \%$ em água destilada durante três minutos (OM) (SPONHOLZ et al., 2004; COSTA; ERABADUPITIYA, 2005).

Após os tratamentos, os frutos foram armazenados em temperatura ambiente e analisados aos um, sete e 14 dias para os parâmetros obtidos de cor da casca por imagem digital, e diariamente para avaliação visual.

\section{Análise de coloração da casca}

Para avaliação utilizando imagens digitais, empregou-se a metodologia descrita por Oliveira et al. (2003). A câmara digital utilizada foi uma Olympus (modelo D-435 U), com resolução de 1600 x 1200 pixels, objetiva de $6,1 \mathrm{~mm}$. A lente da câmara foi posicionada paralela à superfície do produto a uma distância de $20 \mathrm{~cm}$, utilizando $50 \%$ de zoom. Utilizou-se um sistema de iluminação com fonte fluorescente, incidindo com ângulo de $90^{\circ}$ sobre os frutos colocados sobre fundo branco. Foram obtidas imagens digitais em extensão bmp. Para conversão das imagens em valores RGB (red-green-blue) médios, utilizou-se o programa "Conversor em cor média RGB para imagens BMP” (SACHS, 2002). A cor média de cada tratamento foi obtida por meio do programa MICROSOFT®PAINT, versão 5.1, utilizando os valores de RGB. Os valores de RGB foram ainda convertidos para o sistema CIELAB pelo programa "Munsell Conversion versão 4.01", obtendo-se os valores de $\mathrm{L}^{*}$ (luminosidade), a* (componente vermelho-verde), $\mathrm{b}^{*}$ (componente amarelo - azul).

Para avaliação visual utilizou-se a escala de coloração com notas de um a sete segundo proposta do Programa Nacional para Modernização da Agricultura (HORTIBRASIL, 2006), descrita a seguir: 1- Totalmente verde, 2-verde com traços amarelos, 3 - mais verde que amarela, 4 - mais amarela que verde, 5 - amarelo com pontas verdes, 6 - amarelo, 7 - amarelo com pontos marrons, com três repetições por amostra. 


\section{Resultados e Discussão}

Na Tabela 1 estão apresentados os dados da avaliação visual utilizando a escala de notas para bananas submetidas a seis diferentes tratamentos pós-colheita e armazenadas por 14 dias.
$\mathrm{Na}$ Figura 1 encontram-se a cor média obtida a partir dos valores médios de RGB (red-greenblue) de frutos de banana submetidos aos diferentes tratamentos.

Tabela 1. Valores médios da cor da casca de bananas submetidas a seis diferentes tratamentos pós-colheita e armazenadas por 14 dias, segundo escala visual de coloração*.

\begin{tabular}{ccccccc}
\hline Dias & $\mathrm{T}$ & $\mathrm{H} 3$ & $\mathrm{H} 8$ & $\mathrm{HP}$ & $\mathrm{OS}$ & $\mathrm{OM}$ \\
\hline 01 & $1,0( \pm 0)$ & $1,0( \pm 0)$ & $1,0( \pm 0)$ & $1,0( \pm 0)$ & $1,0( \pm 0)$ & $1,0( \pm 0)$ \\
02 & $1,0( \pm 0)$ & $1,0( \pm 0)$ & $1,0( \pm 0)$ & $1,0( \pm 0)$ & $1,0( \pm 0)$ & $1,0( \pm 0)$ \\
03 & $1,0( \pm 0,00)$ & $1,0( \pm 0,00)$ & $1,5( \pm 0,48)$ & $2,0( \pm 0,00)$ & $1,0( \pm 0,00)$ & $1,0( \pm 0,00)$ \\
04 & $2,0( \pm 0,00)$ & $1,5( \pm 0,48)$ & $2,5( \pm 0,48)$ & $3,5( \pm 0,48)$ & $1,0( \pm 0,00)$ & $1,0( \pm 0,00)$ \\
05 & $3,5( \pm 0,48)$ & $2,0( \pm 0,00)$ & $4,0( \pm 0,82)$ & $4,5( \pm 0,48)$ & $2,0( \pm 0,00)$ & $2,0( \pm 0,00)$ \\
06 & $4,5( \pm 0,48)$ & $2,5( \pm 0,48)$ & $5,5( \pm 0,48)$ & $5,5( \pm 0,48)$ & $3,0( \pm 0,00)$ & $2,5( \pm 0,48)$ \\
07 & $5,5( \pm 0,48)$ & $4,5( \pm 048)$ & $6,0( \pm 0,00)$ & $6,0( \pm 0,00)$ & $3,0( \pm 0,00)$ & $2,5( \pm 0,48)$ \\
08 & $5,5( \pm 0,48)$ & $4,5( \pm 048)$ & $6,0( \pm 0,00)$ & $6,0( \pm 0,00)$ & $3,0( \pm 0,00)$ & $3,0( \pm 0,00)$ \\
09 & $6,0( \pm 0,00)$ & $5,0( \pm 0,00)$ & $6,0( \pm 0,00)$ & $6,0( \pm 0,00)$ & $4,0( \pm 0,00)$ & $4,0( \pm 0,00)$ \\
10 & $6,0( \pm 0,00)$ & $6,0( \pm 0,00)$ & $6,0( \pm 0,00)$ & $6,0( \pm 0,00)$ & $4,0( \pm 0,00)$ & $4,0( \pm 0,00)$ \\
11 & $6,0( \pm 0,00)$ & $6,0( \pm 0,00)$ & $6,0( \pm 0,00)$ & $6,0( \pm 0,00)$ & $4,5( \pm 0,48)$ & $4,5( \pm 048)$ \\
12 & $6,5( \pm 048)$ & $6,0( \pm 0,00)$ & $6,5( \pm 048)$ & $6,5( \pm 048)$ & $5,0( \pm 0,00)$ & $5,0( \pm 0,00)$ \\
13 & $6,5( \pm 048)$ & $6,0( \pm 0,00)$ & $6,5( \pm 048)$ & $7,0( \pm 0,00)$ & $5,0( \pm 0,00)$ & $5,0( \pm 0,00)$ \\
14 & $7,0( \pm 0,00)$ & $6,5( \pm 0,00)$ & $7,0( \pm 0,00)$ & $7,0( \pm 0,00)$ & $5,0( \pm 0,00)$ & $5,0( \pm 0,00)$ \\
\hline
\end{tabular}

* média ( \pm desvio padrão)

Escala: 1- Totalmente verde, 2-verde com traços amarelos, 3 - mais verde que amarela, 4 - mais amarela que verde, 5 - amarelo com pontas verdes, 6 - amarelo, 7 - amarelo com pontos marrons

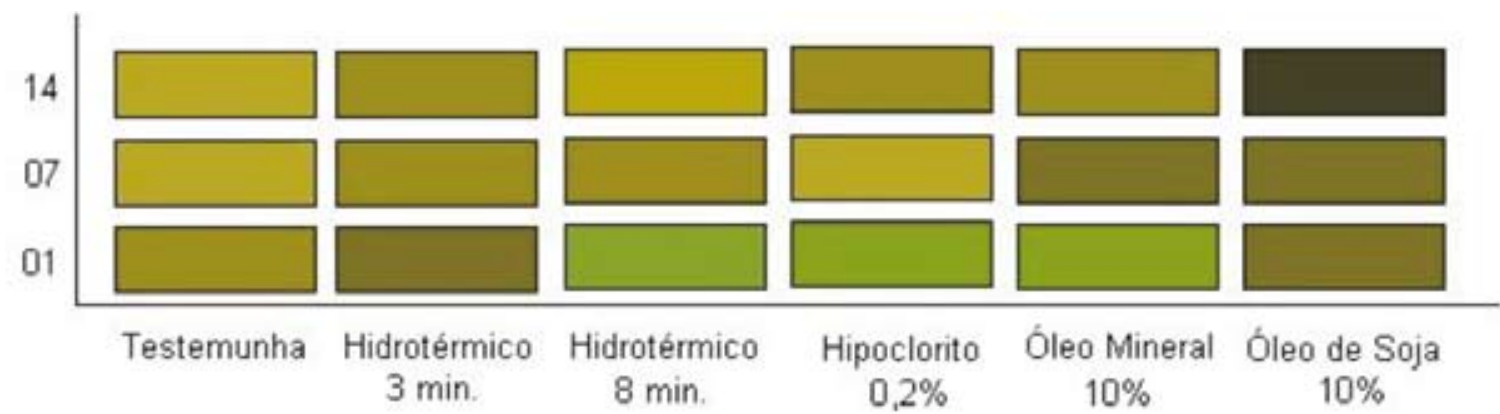

Figura 1. Cor média dos frutos de banana, obtida a partir dos valores de rgb (red-green-blue), para os seis tratamentos pós-colheita durante o período de armazenamento de 14 dias.

No início do experimento, todos os frutos encontravam-se na coloração 01 (totalmente verdes), segundo a escala visual subjetiva de classificação. Como decorrer dotempo, pode-se perceberamudança de coloração gradual do verde para o amarelo na maior parte dos tratamentos, tanto pela escala visual
(Tabela 1), como pelas colorações médias obtidas pelos parâmetros RGB, que representam a mistura das cores vermelho, verde e amarelo (Figura 1). Tal mudança na coloração ocorreu mais rapidamente que a testemunha nos frutos dos tratamentos $\mathrm{H} 8$ (Hidrotérmico $8 \mathrm{~min}$.) e HP (Hipoclorito a $0,2 \%$ ), o 
que sugere que eles não devem ser empregados para controlar o amadurecimento, já que o aspecto visual influencia diretamente na escolha do consumidor (MATSUURA; COSTA; FOLEGATTI, 2004; CHITARRA; CHITARRA, 2005). Em banana, os carotenóides, já estão presentes na casca muito antes de se iniciar o processo de maturação, no entanto, somente com o decorrer do processo de amadurecimento, quando a clorofila é gradualmente destruída pela atividade enzimática, é que a coloração amarela torna-se mais evidente (AWAD, 1993).

Nos tratamentos OS (óleo de soja) e OM (óleo mineral), observou-se um escurecimento e surgimento de manchas na casca a partir do sétimo dia de armazenamento, sendo mais acentuadas no tratamento OS. Este escurecimento também pode ser observado pela coloração média obtida a partir dos valores de RGB, na qual a cor obtida pelas médias do tratamento OS apresentou-se, aos sete dias, mais escura que as demais.

Na Tabela 2 estão apresentados os valores de L* (luminosidade), $\mathrm{a}^{*}$ (componente vermelho-verde), b* (componente amarelo - azul), obtidos por imagem digital, para os frutos de banana.

Tabela 2. Valores de L* (luminosidade), a* (componente vermelho-verde), b* (componente amarelo - azul), obtidos por imagem digital, para os frutos de banana submetidos a seis tratamentos pós-colheita e armazenados por 14 dias*.

\begin{tabular}{cccccccccc}
\hline \multirow{2}{*}{ Trat. } & \multicolumn{3}{c}{$\mathrm{L}^{*}$} & \multicolumn{3}{c}{$\mathrm{a}^{*}$} & \multicolumn{3}{c}{$\mathrm{b}^{*}$} \\
\cline { 2 - 10 } & \multicolumn{2}{c}{ Tempo de armazenamento } & \multicolumn{2}{c}{ Tempo de armazenamento } & \multicolumn{2}{c}{ Tempo de armazenamento } \\
\cline { 2 - 9 } & 01 & 07 & 14 & 01 & 07 & 14 & 01 & 07 & 14 \\
\hline \multirow{2}{*}{$\mathrm{T}$} & 51,24 & 60,93 & 57,28 & $-23,29$ & $-9,13$ & $-3,81$ & 53,72 & 61,68 & 57,80 \\
& $( \pm 0,88)$ & $( \pm 1,66)$ & $( \pm 1,73)$ & $( \pm 2,37)$ & $( \pm 0,24)$ & $( \pm 0,35)$ & $( \pm 1,13)$ & $( \pm 0,73)$ & $( \pm 1,00)$ \\
& 45,03 & 45,92 & 58,94 & $-21,40$ & $-11,57$ & $-7,38$ & 48,20 & 49,33 & 61,53 \\
H3 & $( \pm 0,92)$ & $( \pm 3,64)$ & $( \pm 1,23)$ & $( \pm 0,50)$ & $( \pm 0,81)$ & $( \pm 0,69)$ & $( \pm 3,53)$ & $( \pm 1,14)$ & $( \pm 0,88)$ \\
& 51,12 & 52,74 & 57,70 & $-24,00$ & $-6,27$ & $-3,86$ & 52,08 & 54,27 & 62,17 \\
H8 & $( \pm 0,95)$ & $( \pm 1,10)$ & $( \pm 0,82)$ & $( \pm 1,81)$ & $( \pm 1,12)$ & $( \pm 0,54)$ & $( \pm 0,08)$ & $( \pm 1,51)$ & $( \pm 1,65)$ \\
& 50,23 & 58,61 & 49,29 & $-24,53$ & $-8,90$ & $-3,07$ & 52,97 & 57,86 & 52,90 \\
HP & $( \pm 0,88)$ & $( \pm 0,95)$ & $( \pm 1,21)$ & $( \pm 0,65)$ & $(0,92)$ & $( \pm 0,44)$ & $( \pm 1,06)$ & $( \pm 0,80)$ & $( \pm 0,79)$ \\
& 48,46 & 38,39 & 26,56 & $-22,98$ & $-5,88$ & $-0,31$ & 52,50 & 44,49 & 29,14 \\
OS & $( \pm 0,88)$ & $( \pm 1,26)$ & $( \pm 1,15)$ & $( \pm 1,78)$ & $( \pm 0,69)$ & $( \pm 0,03)$ & $( \pm 2,62)$ & $( \pm 1,59)$ & $( \pm 1,32)$ \\
& 51,11 & 43,27 & 46,15 & $-26,64$ & $-19,42$ & $-4,11$ & 54,73 & 48,95 & 52,22 \\
OM & $( \pm 3,71)$ & $( \pm 0,56)$ & $( \pm 1,03)$ & $( \pm 0,56)$ & $( \pm 1,00)$ & $( \pm 0,35)$ & $( \pm 1,26)$ & $( \pm 0,93)$ & $( \pm 1,07)$ \\
\hline
\end{tabular}

* médias de três repetições

Na pesquisa em alimentos, a cor é comumente representada utilizando o espaço de cor $\mathrm{L}^{*}, \mathrm{a}^{*}$, b*, uma vez que esta representação de cor é a mais associada à percepção humana (MENDOZA; DEJMEK; AGUILERA, 2006). Observou-se aumento da luminosidade durante o armazenamento nos tratamentos $\mathrm{T}, \mathrm{H} 3$ e $\mathrm{H} 8$, o que significa clareamento dos frutos. Porém os tratamentos HP, OS e OM apresentam comportamento inverso, com escurecimento dos frutos (redução de $L^{*}$ ) mais acentuado no tratamento OS, confirmando os dados obtidos pela escala visual e cor média dos frutos.

Em relação ao componente vermelho-verde (a*) observou-se em todos tratamentos, aumento 
dos valores de $\mathrm{a}^{*}$, indicando perda de coloração verde e aumento da cor vermelha. Já para os valores do componente amarelo-azul ( $\left.\mathrm{b}^{*}\right)$, ocorreu aumento nos tratamentos T, H3 e H8 e diminuição nos tratamentos HP, OS e OM, comportamento semelhante à luminosidade. Isto indica aumento da coloração amarela dos frutos para os três primeiro tratamentos e perda de cor amarela e aumento da cor azul para os três tratamentos seguintes, o que também está associado ao escurecimento do produto.

O escurecimento dos frutos pode estar associado à redução da taxa de respiração pela presença de óleo na casca, que pode reduzir a intensidade das trocas gasosas. Isto pode levar ao aumento da concentração de $\mathrm{CO}_{2}$ na superfície da casca, favorecendo a formação de álcoois e aldeídos além de outros compostos, gerando a perda da fluorescência da clorofila e causando o escurecimento de tecidos sensíveis (AWAD, 1993; CHITARRA; CHITARRA, 2005).

Os parâmetros de cor L*, a*, b* apresentaram resultados semelhantes à avaliação da cor por meio da escala visual e cor média obtida pela conversão dos parâmetros de RGB, indicando boa correlação entre os métodos. No entanto, este método, por não utilizar calibração, deve ser utilizado na comparação de dados entre tratamentos testados. Mendoza, Dejmek e Aguilera (2006), avaliando a coloração em bananas com imagens digitais e dados obtidos por colorímetro, observaram boa correlação entre os métodos, sendo que a análise de cor por meio de imagens digitais é um método rápido e fácil de aquisição de dados a um baixo custo.

\section{Conclusão}

Os frutos submetidos aos tratamentos de imersão por 3 minutos em solução aquosa de óleo de soja ou óleo mineral (10\%) apresentaram alterações indesejáveis na coloração da casca, indicando toxidez. Os tratamentos testados promoveram uma evolução gradual da cor dos frutos tratados do verde para o amarelo, porém não ampliaram o tempo de armazenamento do produto.

Os dados obtidos por meio das imagens digitais (convertidos para o sistema CIELAB, e cor média em RGB) confirmaram as observações visuais, indicando uma boa relação entre os métodos.

\section{Referências}

ASSOCIAÇÃO BRASILEIRA DE NORMAS TÉCNICAS - ABNT. Especificações de cores de acordo com o sistema de notação Munsell. NBR 12694. Rio de Janeiro: ABNT, 1992.

AWAD, M. Fisiologia pós-colheita de frutos. São Paulo: Nobel, 1993.

BORGES, M. T. M. R. Potencial vitamínico de banana verde e produtos derivados. 2003. Tese. (Doutorado em Ciência de Alimentos) - Faculdade de Engenharia de Alimentos da Universidade Estadual de Campinas, Campinas.

BOTREL, N.; FREIRE JUNIOR, M.; VASCONCELOS, R. M.; BARBOSA, H. T. G. Inibição do amadurecimento da banana-'Prata-Anã' com a aplicação do 1-metilciclopropeno. Revista Brasileira de Fruticultura, Cruz das Almas, v. 24, n. 1, p. 53-56. 2002.

BRACKMANN, A.; NEUWALD, D. A.; STEFFENS, C. A.; SESTARI, I.; GIEHL, R. F. H. Banana conservada artificialmente. Cultivar Hortaliças e Frutas, Pelotas, v. 5, n. 30, p. 18-21. 2005.

CAMPOS, R. P.; VALENTE, J. P.; PEREIRA, W. E. Conservação pós-colheita de banana nanicão climatizada e comercializada em Cuiabá-MT e região. Revista Brasileira de Fruticultura, Cruz das Almas, v. 25, n. 1, 2003.

CHITARRA, M. I. F.; CHITARRA, A. B. Qualidade póscolheita. In: . Pós-colheita de frutas e hortaliças: fisiologia e manuseio. 2.ed. Lavras: Ed. da UFLA, 2005. p.541-570.

COSTA, D. M.; ERABADUPITIYA, H. R. U. T. An integrated method to control postharvest diseases of banana using a member of the Burkholderia cepacia complex. Postharvest Biology and Technology, Pulmann, v. 36, n. 1, p. 31-39, 2005.

FOOD AND AGRICULTURE ORGANIZATION OF THE UNITED NATIONS - FAO. Faostat database. Agricultural production; agriculture and food trade. Disponível em: <http://www.fao.org $>$. Acesso em: 15 jul. 2006. 
HORTIBRASIL. Programa Brasileiro para Modernização da Horticultura. Banana: cartilha para classificação. Disponível em: <www.hortibrasil.org.br>. Acesso em: 24 set. 2006.

MATSUURA, F. C. A. U.; COSTA, J. I. P.; FOLEGATTI, M. I. S. Marketing de banana: preferências do consumidor quanto aos atributos de qualidade dos frutos. Revista Brasileira de Fruticultura, Cruz das Almas, v. 26, n. 1, p. 48-52, 2004.

MENDOZA, F.; DEJMEK, P.; AGUILERA, J. M. A. Calibrated color measurements of agricultural foods using image analysis. Postharvest Biology and Technology, Pullman, n. 41, p. 285-295, 2006.

OLIVEIRA, A. P. V.; FRASSON, K.; YAMASHITA, F.; BENASSI, M. T. Medida instrumental de cor em sobremesa lácteas de Chocolate: uma técnica de baixo custo e versátil utilizando câmara digital. Brazilian Journal of Food Technology, Campinas, v. 6, n. 2, p. 191196, jul./dez. 2003.

POMERANZ, Y.; MELOAN, C. E. Food analysis: theory and practices. 3.ed. New York: Chapman \& Hall, 1994. p. $87-98$.
SACHS, L. G. Parâmetros agronômicos e tecnológicos para produção e emprego de farinha desengordurada de girassol (Helianthus annus L.) em sistema alimentar. 2002. Tese. (Doutorado em Ciência de Alimentos) Universidade Estadual de Londrina, Londrina.

SACHS, L. G.; PRUDENCIO-FERREIRA, S. H.; PORTUGAL, A. P.; FELINTO, A. S.; SACHS, J. P. D. Leitura de imagem pixel a pixel: I. Uso no controle de qualidade de alimentos. In: SIMPÓSIO LATINO AMERICANO DE CIÊNCIAS DE ALIMENTOS, 4., 2001, Campinas. Anais... Campinas: UNICAMP, 2001. p. 258-258.

SOARES, A. L. Medidas de cor em alimentos e suas aplicações. 2000. Monografia. (Especialização em Ciência de Alimentos) - Universidade Estadual de Londrina, Londrina.

SPONHOLZ, C.; BATISTA, U. G.; ZAMBOLIM, L.; SALOMÃO, L. C. C.; CARDOSO, A. A. Efeito do tratamento hidrotérmico e químico de frutos de banana 'Prata' no controle da antracnose em pós-colheita. Fitopatologia Brasileira, Brasilia, v. 29, n. 5, p. 480-485, 2004. 
